\title{
Josephson current through a precessing classical spin
}

\author{
S Teber ${ }^{1,2}$, C Holmqvist ${ }^{2,3}$, M Houzet ${ }^{4}$, D Feinberg ${ }^{2}$ and M Fogelström ${ }^{3}$ \\ ${ }^{1}$ LPTHE, Universités Paris 6, 7 et CNRS, 4 place Jussieu, 75252 Paris, France \\ ${ }^{2}$ Institut Néel, CNRS and Université Joseph Fourier, BP 166, 38042 Grenoble, France \\ ${ }^{3}$ Department of Microtechnology and Nanoscience, Chalmers 41296, Göteborg, Sweden \\ ${ }^{4}$ INAC/SPSMS, CEA Grenoble 17 rue des Martyrs, 38054 Grenoble Cedex, France
}

\begin{abstract}
A study of the dc Josephson current between two superconducting leads in the presence of a precessing classical spin is presented. The precession gives rise to a time-dependent tunnel potential which not only implies different tunneling probabilities for spin-up and spin-down quasiparticles, but introduces also a time-dependent spin-flip term. We provide an exact general analytic solution for the out-of-equilibrium steady-state permanent current between two spin-singlet superconductors as a function of the superconducting phase difference, the precession frequency and for arbitrary junction transparency. As an application we focus on the effects of the spin-flip term alone and show that the magnitude and nature of the Josephson current are indeed strongly affected by the precession of the classical spin.
\end{abstract}

Contacting a single molecule in a superconducting nanojunction is a challenging goal, especially if the molecule carries a magnetic moment that can precess in presence of a local magnetic field. The effect of a Josephson current on such a precession was considered by Zhu et al. [1]. Here we address the reverse problem, e. g. how the precession of a classical spin inside a junction affects the Josephson current [2]. We assume a spin-dependent tunneling of quasiparticles but contrarily to Ref. [2] address the case of arbitrary transparency. We consider two s-wave superconductors coupled over a precessing classical spin, $\vec{S}$, positioned between them. The precession gives rise to a time-dependent tunneling term

$$
\hat{H}_{T}=\hat{\psi}_{R}^{\dagger} \hat{T}_{R L}(t) \hat{\psi}_{L}+H . C .,
$$

Preprint submitted to Physica B

October 22, 2010 
in the Hamiltonian where $\hat{\psi}_{\alpha}^{\dagger}=\left(c_{\alpha, k, \uparrow}^{\dagger}, c_{\alpha,-k, \downarrow}, c_{\alpha,-k, \uparrow}, c_{\alpha, k, \downarrow}^{\dagger}\right)$ is the Nambuspinor in lead $\alpha=\mathrm{R}, \mathrm{L}$ and $c$ is a fermionic annihilation operator. The hopping matrix $\hat{T}_{R L}(t)\left(=\hat{T}_{L R}^{\dagger}(t)\right)$ has a spin-structure. It may be parametrized into a spin-independent amplitude $T_{o}$ and a spin-dependent part $T_{s}\left(\cos \vartheta \vec{S}_{\|}+\right.$ $\left.\sin \vartheta \vec{S}_{\perp}(t)\right) \cdot \vec{\sigma}$ corresponding to a Kondo-like coupling between the classical spin and the spin of the conduction electrons of the leads. The spinquantization axis for the tunneling quasiparticles is given by the precession axis, $\vec{S}_{\|}$, and $\vec{S}_{\perp}(t)$ gives the instantaneous projection of the precessing spin in the plane. The spin-independent amplitude together with the parallel portion of the spin-matrix, $T_{o}+T_{s} \cos \vartheta \vec{S}_{\|} \cdot \vec{\sigma}$, causes a difference in tunneling amplitude for spin-up and spin-down quasiparticles, while the perpendicular portion, $\sin \vartheta \vec{S}_{\perp}(t)$, induces spin flips. The latter is time-dependent. For a precession frequency $\Omega$, the hopping matrix in combined $\operatorname{spin} \otimes$ Nambu space reads:

$$
\hat{T}_{R L}(t)=\left(\begin{array}{cc}
\sigma_{z} T_{o}+T_{\|} & i \sigma_{y} T_{\perp} e^{-i \Omega t} \\
-i \sigma_{y} T_{\perp} e^{i \Omega t} & -\sigma_{z} T_{o}-T_{\|}
\end{array}\right),
$$

where the $\sigma_{j}$ are usual Pauli matrices, $T_{\|}=T_{s} S \cos \vartheta$ and $T_{\perp}=T_{s} S \sin \vartheta$. In this simple model the tunneling amplitudes are assumed to be energyindependent and we will also neglect the external source giving rise to, and maintaining, the precession of the classical spin.

In order to calculate the Josephson current through the spin we need to solve the time-dependent boundary conditions imposed by $\hat{H}_{T}$. In Ref. [3] this was done by means of the quasiclassical formulation of the T-matrix method. Here, we provide the interested reader with an approach based on the quasiclassical out-of-equilibrium Green's function technique [4]. With the help of this formalism, the Josephson current flowing through the junction reads:

$$
I=e \operatorname{Tr}\left[\hat{\sigma}_{z} \hat{T}_{R L}(t) \hat{G}_{L R}^{-}(t, t)\right],
$$

where the current is computed from the right side and we have dropped the lead index, $\hat{G}_{L R}^{-}\left(t, t^{\prime}\right)$ is the lesser Keldysh Green's function in spin $\otimes$ Nambu space and $\hat{\sigma}_{z}$ is the corresponding Pauli matrix. In the present dc case, the expression for this Green's function is quite simple and reads:

$$
\begin{aligned}
\hat{G}_{L R}^{-}\left(t, t^{\prime}\right)= & -\left(\hat{G}_{L R}^{R} \circ n_{F}-n_{F} \circ \hat{G}_{L R}^{A}\right)\left(t, t^{\prime}\right)+ \\
& +\hat{G}_{L R}^{R} \circ\left(\hat{T}_{R L} \circ n_{F}-n_{F} \circ \hat{T}_{R L}\right) \circ \hat{G}_{L R}^{A}\left(t, t^{\prime}\right) \\
& +\hat{G}_{L L}^{R} \circ\left(\hat{T}_{L R} \circ n_{F}-n_{F} \circ \hat{T}_{L R}\right) \circ \hat{G}_{R R}^{A}\left(t, t^{\prime}\right),
\end{aligned}
$$


where $\circ$ is a time convolution and $n_{F}$ is the Fermi occupation function which is the same in both leads. In Eq. (田) the dressed spectral functions satisfy the usual Dyson equations:

$$
\begin{aligned}
& \hat{G}_{L R}^{(R, A)}\left(t, t^{\prime}\right)=\hat{g}_{L}^{(R, A)} \circ \hat{T}_{L R} \circ \hat{g}_{R}^{(R, A)}\left(t, t^{\prime}\right)+\hat{g}_{L}^{(R, A)} \circ \hat{T}_{L R} \circ \hat{g}_{R}^{(R, A)} \circ \hat{T}_{R L} \circ \hat{G}_{L R}^{(R, A)}\left(t, t^{\prime}\right), \\
& \hat{G}_{L L}^{(R, A)}\left(t, t^{\prime}\right)=\hat{g}_{L}^{(R, A)}\left(t-t^{\prime}\right)+\hat{g}_{L}^{(R, A)} \circ \hat{T}_{L R} \circ \hat{g}_{R}^{(R, A)} \circ \hat{T}_{R L} \circ \hat{G}_{L L}^{(R, A)}\left(t, t^{\prime}\right),
\end{aligned}
$$

where $\hat{g}$ are the local Green's functions of the disconnected leads and the Dyson equation for $\hat{G}_{R R}$ may be obtained from the one of $\hat{G}_{L L}$ by interchanging left and right indices.

In order to proceed further we gauge out the precession frequency from the tunneling Hamiltonian of Eq. (11) with the help of the following time- and spin-dependent unitary transformation:

$$
U_{\alpha, k, \sigma}(t)=\exp \left(-i \frac{\sigma \Omega t}{2} c_{\alpha, k, \sigma}^{\dagger} c_{\alpha, k, \sigma}\right)
$$

where $\sigma= \pm$ is the spin index. In the rotating frame, the Hamiltonian $\hat{H}$ of the junction becomes time-independent:

$$
\hat{\tilde{H}}=\hat{H}(\Omega=0)-\sum_{\alpha} \hat{\tilde{\psi}}_{\alpha}^{\dagger} \hat{h}_{z} \hat{\tilde{\psi}}_{\alpha}
$$

where the $\hat{\tilde{\psi}}_{\text {s }}$ are rotated spin-Nambu Grassmann fields and $\hat{h}_{z}$ is an effective magnetic field in spin $\otimes$ Nambu space. The latter has an amplitude: $h_{z}=\Omega / 2$ and shifts the energy of spin-up and down quasiparticles in the leads by $\pm h_{z}$, respectively. The time-dependent problem we consider therefore has a stationary solution which allows us to go to Fourier space. Moreover, not only does $h_{z}$ affect the spectral Green's functions as a usual magnetic field would do: $\hat{\tilde{g}}^{(R, A)}(\omega)=\hat{g}^{(R, A)}\left(\omega+\hat{h}_{z}\right)$. It also, and contrary now to the action of a usual magnetic field, affects the Keldysh Green's function and therefore the occupation functions of the leads which acquire a matrix-structure in spinNambu: $\hat{\tilde{n}}_{F}(\omega)=n_{F}\left(\omega+\hat{h}_{z}\right)$, similarly for both leads. This subtle action of $h_{z}$ on the occupation functions of the leads translates the out-of-equilibrium nature of the problem in the rotating frame. Applying the unitary transformation to the gauge-invariant current of Eq. (3) the problem reduces to solve a set of equations, Eqs. (4) and (5), which are now algebraic. In the rotating frame, we therefore face a time-independent problem of non-colinear magnetization consisting of a static tilted spin in the junction together with an 
effective, $z$-directed, magnetic-field acting on the spectral and occupation functions of the leads. This mapping is valid to all orders in the precession frequency, the transparency of the junction and for arbitrary orientations of the spin.

As an application we will focus now on how the Josephson current is affected by spin flips only and let the spin direction rotate with an angular frequency $\Omega$ in the plane, thus setting $T_{o}=0$ and $\vartheta=\pi / 2$ in the rest of the paper. This case is not only of academic interest as large tilt angles may be reached experimentally in the case of single-molecule magnets (SMM). This is in contrast, e.g. to the case of an SFS junction under an external radiation [5] where the tilt angle is small.

We proceed in analyzing the current-carrying processes across the localized spin first focusing on the equilibrium case, i.e. $\Omega=0$. In this limit the current in Eq. (3) has a rather simple form and we can write down the current-phase relation at arbitrary transparency, $\mathcal{D}=4 t_{\perp}^{2} /\left(1+t_{\perp}^{2}\right)^{2}$ where $t_{\perp}=T_{\perp} / W$ ( $W$ being the band-width), and temperature, $T$, as

$$
I(\varphi)=-\frac{e}{2 \hbar} \Delta \frac{\mathcal{D} \sin \varphi}{\sqrt{1-\mathcal{D} \cos ^{2} \frac{\varphi}{2}}} \tanh \frac{\varepsilon_{J}(\varphi)}{2 T}, \quad \Omega=0 .
$$

This expression is identical to the one without spin flips [7] up to an extra phase shift of $\pi$. The current is therefore carried by Andreev-bound states below the superconducting gap with the phase dispersion $\varepsilon_{J}(\varphi)=$ $\pm \Delta \sqrt{1-\mathcal{D} \cos ^{2} \frac{\varphi}{2}}$

As a second limiting case we consider the case of finite precession frequency in the tunnel limit, i.e. $t_{\perp} \rightarrow 0$. The Josephson current then changes into a steady-state non-equilibrium current. However, it still has a rather simple analytic form at $T=0$ :

$$
I(\varphi)=-\frac{e}{\hbar} \Delta \frac{4 t_{\perp}^{2} \sin \varphi}{\pi} f\left(\frac{\Omega}{2 \Delta}\right), \quad t_{\perp} \rightarrow 0,
$$

where: $f(x)=K(x)$ for $x<1$ while $f(x)=K(1 / x) / x$ for $x>1$ and $K$ is the complete elliptic integral of the first kind. At small precessing frequencies: $K(\Omega / 2 \Delta) \approx \pi / 2+(\pi / 8)(\Omega / 2 \Delta)^{2}+\ldots$. We therefore recover Eq. (8) as a limiting case with first corrections quadratic in $\Omega$. Indeed, in order to transfer a Cooper pair from one lead to another, an Andreev spin-down hole will flip its spin absorbing a quantum of precession while, simultaneously, an Andreev spin-up electron will flip its spin emitting a quantum of precession 
(vice-versa for $\Omega<0$ ). On the other hand, in the limit $\Omega \rightarrow 2 \Delta$, the current in Eq. (9) diverges logarithmically. As known in the field of SNS junctions, see e.g. Ref. [6], such a non-analyticity translates the fact that this current is carried by extended states above the superconducting gap. The divergence may be cured either by considering finite temperatures or by resuming the perturbation theory in $t_{\perp}$.

Within such a finite-temperature re-summed theory we face a more subtle regime where both bound-states localized below the gap as well as extended states above it carry the current. The expression of the latter is more complicated but may still be written in a compact form as

$$
\begin{aligned}
& I(\varphi)=-\frac{e}{2 \hbar} \tilde{\mathcal{D}} \sin \varphi \operatorname{Im} \int \frac{d \varepsilon}{2 \pi} \frac{\left[\phi_{-}-\phi_{+}\right] \lambda_{1}+\left[\phi_{-}+\phi_{+}\right] \lambda_{2}}{D^{R} D^{A}}, \\
& \lambda_{1}=\sin \theta_{-}^{R} \sin \theta_{+}^{A}\left(1+\tilde{\mathcal{D}} \cos \theta_{+}^{R} \cos \theta_{-}^{A}\right)-\sin \theta_{+}^{R} \sin \theta_{-}^{A}\left(1+\tilde{\mathcal{D}} \cos \theta_{-}^{R} \cos \theta_{+}^{A}\right), \\
& \lambda_{2}=\sin \theta_{-}^{R} \sin \theta_{+}^{R}\left(1+\tilde{\mathcal{D}} \cos \theta_{+}^{A} \cos \theta_{-}^{A}\right)-\sin \theta_{+}^{A} \sin \theta_{-}^{A}\left(1+\tilde{\mathcal{D}} \cos \theta_{-}^{R} \cos \theta_{+}^{R}\right), \\
& D^{(R, A)}=1+\tilde{\mathcal{D}} \cos \theta_{-}^{(R, A)} \cos \theta_{+}^{(R, A)}-\tilde{\mathcal{D}} \cos \varphi \sin \theta_{-}^{(R, A)} \sin \theta_{+}^{(R, A)}
\end{aligned}
$$

where $\phi_{ \pm}=\tanh \left(\left(\varepsilon \pm h_{z}\right) / 2 T\right), \sin \theta_{ \pm}=\Delta / \sqrt{\Delta^{2}-\left(\varepsilon \pm h_{z}\right)^{2}}, \cos \theta_{ \pm}=-i(\varepsilon \pm$ $\left.h_{z}\right) / \sqrt{\Delta^{2}-\left(\varepsilon \pm h_{z}\right)^{2}}$ and the retarded and advanced functions correspond to: $\varepsilon \rightarrow \varepsilon \pm i 0^{+}$. For convenience we have redefined the transparency as: $\tilde{\mathcal{D}}=2 t_{\perp}^{2} /\left(1+t_{\perp}^{4}\right)$.

Eq. (10) is the central formula of this paper. It has both Eqs. (8) and (9) as limiting cases. We clearly see in Eq. (10) the out-of-equilibrium effect of $h_{z}$ at the level of the occupation functions. Moreover, Eq. (10) has both branchcuts over a width $\pm h_{z}$ around $\pm \Delta$ which correspond to current-carrying extended states, and poles, $\varepsilon_{J}(\varphi)$, corresponding to current-carrying boundstates. Extended- and bound-states are inter-related as can be seen from the spectrum of the latter which differs considerably from the equilibrium case:

$\varepsilon_{J}(\varphi)= \pm \sqrt{h_{z}^{2}+\frac{\Delta^{2}}{1-\tilde{\mathcal{D}}^{2}}\left\{1+\tilde{\mathcal{D}}^{2} \cos \varphi-\sqrt{\tilde{\mathcal{D}}^{2}(1+\cos \varphi)^{2}+4\left(1-\tilde{\mathcal{D}}^{2}\right)\left(\frac{h_{z}}{\Delta}\right)^{2}}\right\}}$

The current-carrying bound-states merge with the extended states at a phase difference $\varphi_{c}$ such that: $\varepsilon_{J}(\varphi)^{\prime}=0$. Hence, they exist for phase differences smaller than $\varphi_{c}$ and larger than $2 \pi-\varphi_{c}$ where, e.g. for $\tilde{\mathcal{D}}=1$ the phase $\varphi_{c}$ is related to the effective magnetic field by: $h_{z}=\Delta \cos ^{2}\left(\varphi_{c} / 2\right)$. For other 

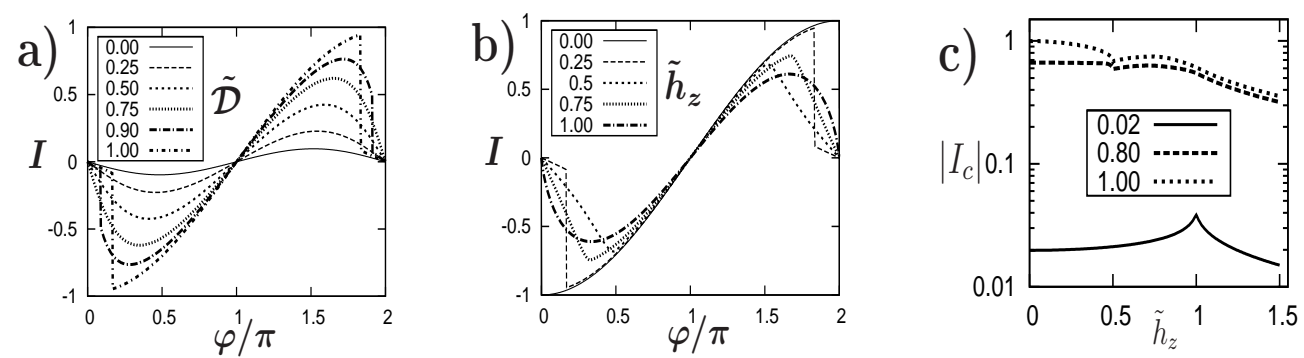

Figure 1: (a) Current-phase relation for a given: $h_{z}=0.25 \Delta$. The different line styles refer to different transparencies, $\tilde{\mathcal{D}}$, see the inset. (b) Current-phase relation for a given: $\tilde{\mathcal{D}}=1$, and different reduced effective magnetic fields, $\tilde{h}_{z}=h_{z} / \Delta$, in units of $\Delta$, see the inset. (c) Absolute value of the critical current, i.e. maximum current as a function of the phasedifference for a given value of $h_{z}$, in logarithmic scale and for different transparencies, $\tilde{\mathcal{D}}$, see the inset. The current is in units of $e \Delta / \hbar$.

phase differences the extended states carry all the current. Fig. 11 displays a numerical evaluation of the current given by Eq. (10). Fig. 1 c) shows that the logarithmic singularity at the $2 \Delta$-resonance, which is related to extended states, has been cured. Moreover, Figs. 1 a) and 1 b) display a low precession frequency $(\Omega \ll 2 \Delta)$ structure consisting of steps with a noticeable variation of the current. These steps correspond to an abrupt change in the occupation of the lower and upper Andreev-levels the currents of which cancel each-other when they are both occupied, see also Ref. [3].

Acknowledgement. Financial support from the Swedish Research Council (VR) is gratefully acknowledged.

[1] J.-X. Zhu et al., Phys. Rev. Lett. 92, 107001 (2004)

[2] J.-X. Zhu, A.V. Balatsky, Phys. Rev. B 67, 174505 (2003)

[3] C. Holmqvist et al., LT25 Proceedings (2008).

[4] J. C. Cuevas et al., Phys. Rev. B 54, 7366 (1996).

[5] M. Houzet, Phys. Rev. Lett. 101, 057009 (2008).

[6] A. Levchenko et al., Phys. Rev. B 74212509 (2006).

[7] C. W. J. Beenakker, Phys. Rev. Lett. 67, 3836 (1991) 